identification, healthcare-worker screening and follow-up after exposure, and engineering controls, eg, ventilation in isolation and treatment rooms. In addition, the directive provides a summary of the new requirements for respiratory protection that include the use of N95 respirators certified under the revised NIOSH certification procedures and requirements for policies for reuse of disposable respirators. Most of the directive reflects recommendations from the $\mathrm{CDC}$. There are a few components that go beyond the $\mathrm{CDC}$ requirements, such as the requirement for two-step PPD baseline skin testing on all employees. Also, there is a requirement for isolation room signage to include (1) the need for a warning sign (eg, "STOP," "HALT," or biological hazard symbol); (2) isolation sign (eg, “AFB" or "respiratory isolation"); and, (3) a description of the necessary precautions to use (eg, don respirators before entering).

Inspections will be conducted in response to employee complaints, fatalities, or as part of a routine industrial hygiene inspection in workplaces that have been identified as having workers with a greater risk of TB than the general population. These include healthcare facilities, correctional institutions, long-term-care facilities, homeless shelters, and drug treatment centers. OSHA states that healthcare facilities include hospitals where patients with confirmed or suspected TB are treated or to which they are transported. Coverage of nonhospital healthcare settings (ie, doctors' offices, clinics, etc) include only personnel present during the performance of high-hazard procedures on suspect or active TB patients. Dental healthcare personnel are covered by this directive only if they treat suspect or active TB patients in a hospital or correctional facility.

This directive provides OSHA's enforcement procedures for TB until a final TB standard is complete. It is expected that the proposed TB standard will be published in the Federal Register for public comment by the end of this year. Copies of the document are available on the Internet at http://www.osha-slc.gov.

FROM: US Department of Labor: OSHA Office of Health Compliance. Enforcement Procedures and Scheduling for Occupational Exposure to Tuberculosis. (OSHA Instruction CPL 2.106) February 9, 1996.

\section{New York City Strain of MDR-TB Spreads to Four States}

Pablo Bifani of the TB Center of the New York Public Health Research Institute and colleagues recently conducted a study to determine whether isolates of Mycobacterium tuberculosis from New York City and elsewhere that are resistant to four or more primary antimicrobial agents and responsible for widespread disease in the 1990s represent a newly emerged clone or a heterogeneous array of unrelated organisms.

Multidrug-resistant tuberculosis (MDR-TB) isolates were recovered from 253 New York City patients and had the same or closely related fingerprint and hybridization patterns, polymerase chain reaction profiles, and DNA gene sequencing containing mutations associated with resistance to rifampin, isoniazid, and streptomycin. These strains were arbitrarily designated as "W" strains. MDR-TB isolates with these same molecular characteristics were recovered from patients in Florida and Nevada, healthcare workers in Atlanta, Georgia, and Miami, Florida, and an individual who had moved from New York City to Denver, Colorado; and caused disease or skin-test conversion in at least 12 people in a nursing home environment.

The authors conclude that their findings document the molecular origin and spread of a closely related family of MDR-TB strains that have shared a common ancestor and undergone clonal expansion. Dissemination of these difficult-to-treat bacteria throughout New York City, and to at least four additional US cities, has adverse implications for TB control in the 21st century.

Although there has been a $21 \%$ decrease in the number of TB cases reported in New York City between 1992 and 1994, several factors suggest that the long-term ability to eradicate these multidrug-resistant strains will be difficult. First, one study documented 32 healthcare workers (HCWs) who became skin-test positive during an outbreak caused by a W strain. If all HCWs are immunocompetent, $5 \%$ of these individuals will develop active TB in their lifetimes. Second, the strain has spread to at least three major US cities (and four states) that have a combined population of 7.1 million and are hubs for air and auto travel. Third, there are no new primary anti-TB medications introduced since the 1960 s, and it is unlikely that there will be any new drugs. Moreover, even successful, directly observed therapy programs are compromised with MDR-TB.

FROM: Bifani PJ, Plikaytis BB, Kapur V, et al. Origin and interstate spread of a New York City MDR-TB clone family. JAMA 1996;275:452-457.

\section{FDA's Final Medical Device Reporting Regulations}

FDA has issued the final Medical Device Reporting regulation for reporting adverse events to manufacturers. It will be effective on April 11, 1996. This regulation is the last component of the Safe Medical Device Act of 1990, giving the FDA the authority to require manufacturers, distributors, and device-user facilities to submit reports to the FDA on certain types of medical-device-related adverse events.

The Medical Device Reporting regulation provides mechanisms for FDA and manufacturers to identify and monitor significant adverse events involving medical devices, so that problems may be detected and corrected in a timely manner. Under this regulation, medical facilities (referred to as device-user facilities) are required to report to the device manufacturer when a device has or may have caused or contributed to a patient death or serious injury. In the case of death or serious injury, user facilities also must send a copy of the report to the FDA.

In addition to individual device reports, medical facilities also must submit semiannual reports to the FDA, establish files related to reportable events, and maintain those files for 2 years. Further, medical facilities must 
establish written procedures for reporting adverse medical device events that include (1) procedures for timely and effective identification and evaluation of event; (2) a standardized review process and procedure for determining whether events are reportable; (3) procedures to assure the timely submission of complete reports; and (4) a mechanism to assure compliance with documentation and recordkeeping requirements.

"Because interim regulations for reporting have been in place since 1990, most healthcare facilities have already developed a reporting program," said Britton Berek, of the American Society for Healthcare Engineering of the American Hospital Association, who has been monitoring the Safe Medical Device Act closely since 1990.

Berek also noted, "The final rule is very similar to the interim proposed rules, however, some changes will need to be made to the existing program." These changes will include expansion of the reporting criteria to include "user error" and "tightening" of the reporting time frame to be within 10 days of the event. In addition, almost all medical facilities are now included, except physician and dental offices.

FDA regulations and guidance documents are available via the Internet at http://www.fda.gov.

FROM: FDA. Medical device reporting. Federal Register December 11, 1995;60:63578.

\section{OSHA's Occupational Injury Reporting}

OSHA announced that it will begin collecting injury and illness data from 80,000 employers in high-hazard industries. At the same time, OSHA has issued proposed changes to the current procedures for recording and reporting workplace injuries, illnesses, and deaths to simplify the system and to improve future use of the data.

In February 1996, injury and illness report forms were scheduled to be mailed to individual workplaces, requesting data from 1995 . The data will be used to expand on a safety and health pilot program being tested in Maine. Assistant Secretary of Labor for Occupational Safety and Health Administration Joseph A. Dear said, "These steps are an important part of OSHA's reinvention efforts to become a data-driven, results-oriented organization.

On February 2, 1996, OSHA published the proposed revision to the "Recording and Reporting Occupational Injuries and Illnesses” (29 CFR:1904). This revision is expected to simplify the reporting process for employers and to improve the quality and accuracy of the safety and health information available for OSHA. In addition, this revision is expected to increase the use of modern technology, including computers, and improved employee awareness and involvement. OSHA estimates that it will reduce the regulatory burden by $\$ 4.7$ million. OSHA has incorporated comments from labor, industry, trade and professional associations (including infection control and hospital groups), and other governmental agencies into the proposed rule.

Under the proposed revisions, healthcare employers would be required to record all bloodborne pathogen exposure incidents that result in disease. Further, OSHA is proposing that employers record lacerations or puncture wounds, eg, needlesticks. OSHA has asked for comments on whether it is appropriate to record these lacerations or puncture wounds if they do not lead to disease. Concern has been expressed already by the healthcare community regarding a requirement for recording bloodborne "exposure incidents" and the unnecessary duplication for those facilities already collecting this information.

OSHA also is proposing that all TB exposures that result in infection (skin-test conversion) be recorded unless there is evidence of a nonwork exposure, such as household or community contact. This proposal is consistent with OSHA's current enforcement procedures for occupational exposure to TB.

Written comments on the proposal must be received by April 29, 1996, in the Docket Office, Docket No. R-02, Room N2625, US Department of Labor, 200 Constitution Ave NW, Washington DC, 20210; telephone 202-219-7894. The text also is on the Internet at http://www.osha.gov in the "What's New Section."

FROM: Department of Labor: OSHA. Occupational injury and illness recording and reporting requirements. Federal Register February 2, 1996;60:4030-4067.

\section{Molecular Epidemiology Workshop}

The CDC, in collaboration with the National French Academy for Basic Research, will sponsor a 3-day workshop on molecular epidemiology and evolutionary genetics of pathogenic microorganisms on June 17-19, 1996, in Atlanta. The purpose of the workshop is to bring together clinicians, epidemiologists, molecular biologists, and evolutionary geneticists working on infectious disease. Healthcare providers, public health professionals, and laboratory scientists are facing a range of issues in combating infectious diseases. There is a critical need to be able to identify infectious agents rapidly in a manner that allows discrimination of closely related strains and species and to use genetic information for studying evolution, emergence, and spread of the pathogens.

For information about the workshop, contact Dr. Michael Tibayrene, telephone 770-488-4524; fax 770-4884454; e-mail: mdt3ciddpd2.em.cdc..gov.

Additional news items in this issue: OSHA Loses Respirator Case, page 285; New TB Test Approved, page 327.

Additional news items in this issue: OSHA Loses Respirator Case, page 285; Simultaneous Influenza and Pneumococcal Vaccines, page 327; New TB Test Approved, page 327. 